\title{
Sensores de corrosão para monitoramento de pontes e viadutos de concreto armado. Primeira etapa - Testes em solução aquosa.
}

\author{
Analiet Calvo Valdés ${ }^{1}$ (iD) orcid.org/0000-0001-8717-3358 \\ Polyana de Jesus Roque ${ }^{1}$ (D) orcid.org/0000-0001-7601-3228 \\ Marcelo Henrique Farias Medeiros ${ }^{1}$ (D) orcid.org/0000-0003-3112-9715 \\ ${ }^{1}$ Universidade Federal do Paraná, Curitiba, Brasil. \\ E-mail do autor principal: Analiet Calvo Valdés, analietcv@yahoo.es
}

\begin{abstract}
Resumo
A utilização de sensores embebidos no concreto para monitorar a durabilidade das estruturas é uma tendência atual. Uns dos dispositivos mais simples achados no mercado são os sensores galvânicos; formados por dois metais dissimilares. Esses sensores de corrosão se baseiam na identificação prematura da variação da corrente galvânica de modo que a evitar degradações que levem ao colapso limitando o período de vida útil do parque de obras de arte da infraestrutura pública. O objetivo do trabalho foi confeccionar um sensor galvânico de múltiplos elétrodos nos laboratórios da UFPR inspirado num equipamento existente no mercado estrangeiro e avaliar o desempenho do mesmo em uma solução saturada de $\mathrm{Ca}(\mathrm{OH})_{2} \mathrm{com}$ e sem adição de $\mathrm{NaCl}$ (3,5\% em massa). Em ambas as condições de exposição foi medida a corrente galvânica e a diferença de potencial no sensor. A corrente ficou da ordem de micros amperes mesmo após a adição de cloreto à solução aquosa. Entretanto, os valores de potencial dos pares se tornaram positivos e com tendência a aumentar. Esperava-se que o sensor se comportasse como um semi-eletrodo de referência, porém, cada par mostrou um comportamento diferenciado com variação maior do que $1 \mathrm{mV} / \mathrm{min}$ entre leituras.
\end{abstract}

Palavras-chave: Sensor; Corrente galvânica; Diferença de potencial.

\begin{abstract}
The use of sensors embedded in concrete to monitor the durability of structures is a current trend. Some of the simplest devices on the market are galvanic sensors; Formed by two dissimilar metals. These corrosion sensors are based on the premature identification of galvanic current variation so as to avoid degradations leading to collapse by limiting the lifetime of the works of art of the public infrastructure. The objective of this work was to prepare a multi-electrode electrode in the laboratories of UFPR inspired by an equipment in the foreign market and to evaluate its performance in a saturated solution of $\mathrm{Ca}(\mathrm{OH}) 2$ with and without addition of $\mathrm{NaCl}$ (3.5\% in large scale). In both conditions of exposure, the galvanic current and the potential difference in the sensor were measured. The current was of the order of micro-amps even after addition of chloride to the aqueous solution. However, the potential values of the pairs became positive and tended to increase. The sensor was expected to behave as a reference semi-electrode, however, each pair showed a different behavior with variation greater than $1 \mathrm{mV} / \mathrm{min}$ between readings.
\end{abstract}

Key-words: Sensor; Galvanic current; Potential difference. 


\section{Introdução}

A preservação das obras de arte da infraestrutura pública (OAEs) pode ser gerida de maneira mais sustentável e inteligente através do acolhimento das inovações, resultados de pesquisas e monitoramento do estado estrutural das OAEs [1]. As ações executadas nas OAEs para prevenir, eliminar ou diminuir a deterioração da estrutura como um todo ou de algum elemento estrutural nela, devem estar enfocadas na eliminação da necessidade de grandes investimentos em obras de reconstrução ou reposição, quando as OAEs ainda estão em boas condições estruturais e de funcionalidade, antes da aparição de danos sérios.

Nesse contexto, o monitoramento do estado estrutural das OAEs tem vindo a suscitar 0 interesse crescente de projetistas e responsáveis pela gestão do parque de obras de arte especiais. Os requisitos para esta atividade de manutenção têm aumentado nas últimas décadas, chegando a incluir questões associadas com a durabilidade dos materiais e das estruturas [2].

Um sistema de monitoramento contínuo baseado em simples de controles periódicos visuais pode detectar deteriorações apenas em estágios bastante avançados, quando as fissuras, manchas de corrosão, desplacamentos de cobrimentos e deformações excessivas das estruturas já estão presentes nos elementos estruturais [3]. Entretanto um sistema de monitoramento que integre inspeções visuais, investigação detalhada e sensores permite detectar de forma precoce o risco de corrosão nas armaduras entre outras patologias. Desta forma as ações de manutenção ou sistemas de proteção podem ser programadas de modo a se gastar menos recursos evitando intervenções mais complexas e caras. Além de admitir a previsão do desempenho futuro da estrutura e tomar decisões quanto ao tempo ideal para fazer uma intervenção.

Para o monitoramento da corrosão em pontes, por exemplo, são necessários sensores embebidos no concreto que medem parâmetros relevantes para a durabilidade das armaduras, designadamente: potencial de corrosão; resistividade do concreto; corrente galvânica e resistência à polarização [2].

Esses sensores para o monitoramento do risco de corrosão em estruturas novas, são posicionados sobre as armaduras antes da concretagem e em estruturas existentes são introduzidos em furos realizados ao longo da espessura do concreto de cobrimento da armadura. Ao longo do tempo, os sensores além de servirem para monitorar o risco de corrosão, podem ser usados para medir a taxa de corrosão do aço, avaliando a eficiência e durabilidade de reparos.

Os sensores podem ser de leitura manual ou automática. No primeiro caso, as leituras são feitas em campo, diretamente nos sensores cujos terminais são conectados a um painel individual ou a um painel central. No segundo caso, as leituras são feitas por meio de sistema eletrônico (software e hardware), podendo os dados ser disponibilizados por sistema de transmissão remota, o que permite $o$ monitoramento da estrutura em tempo real [4]. Isto é uma vantagem com relação às técnicas eletroquímicas usadas em laboratório.

A maioria dos sensores disponíveis no mercado internacional para avaliação do risco da corrosão em estruturas de concreto fundamenta-se no monitoramento da variação da corrente galvânica [5] em uma macrocélula formada por dois metais com potenciais diferentes, colocados em contato através de um eletrólito, por exemplo o concreto [6] o que forma uma célula galvânica.

Os componentes de uma célula galvânica, pilha ou bateria são: (i) os compartimentos: as duas metades da célula; (ii) os eletrodos: superfícies onde ocorrem as reações; (iii) as semi-pilhas: cada eletrodo e o meio onde está imerso; (iv) o circuito externo: circuito elétrico que conecta os eletrodos e permite o escoamento de elétrons através do circuito [7]. Na prática, para a avaliação do risco de corrosão nas estruturas de concreto, os sensores galvânicos consistem de um conjunto de barras de aço carbono, 
eletricamente isoladas. Com o embutimento do sensor no concreto, essas barras ficam posicionadas em diferentes profundidades, sempre menores do que a da armadura. Esse conjunto de barras do sensor é também denominado de ânodo. $\mathrm{Na}$ proximidade do ânodo, é embutida a mencionada barra de metal mais nobre, denominada de cátodo [5].

Esse método de monitoramento utiliza um amperímetro de resistência zero, conhecido também, como técnica ZeroResistance Ammeter (ZRA) [8] para o monitoramento da corrente galvânica. Em concreto íntegro, essa corrente é desprezível, ou seja, muito baixa. Isso porque as barras de aço-carbono estão em estado passivo, portanto, apresentam uma diferença de potencial pequena e estável em relação ao cátodo também passivo. 0 mesmo não ocorre quando um processo corrosivo é estabelecido nas barras. Nesse caso, a corrente galvânica apresenta um valor significativo em decorrência da variação do potencial do ânodo, que assume valores mais negativos em relação ao obtidos inicialmente, em seu estado passivo [5; 8].

O objetivo deste trabalho foi avaliar o desempenho de um sensor galvânico confeccionado nos laboratórios da Universidade Federal do Paraná (UFPR), com com materiais brasileiros e inspirado no Sensor de múltiplos elétrodos (CorroWatch Multisensor), da empresa Force Technology. A estabilidade eletroquímica deste sensor foi testada em ambientes de concreto representativos, a saber, solução saturada de hidróxido de cálcio com e sem adição de $\mathrm{NaCl}$ (3,5\% em massa) simulando contaminação por cloretos presentes na água do mar.

\section{Configuração do sensor}

A macro célula galvânica considerada neste estudo é formada por cobre e aço carbono dispostos num formato semelhante ao sensor galvânico CorroWhatch da Force Technology. Foram usadas barras de aço-carbono de $60 \mathrm{~mm}$ de comprimento e diâmetro 8-10 mm (ânodo) a diferentes alturas e instalados sobre uma placa de cobre (cátodo) de 50 por $50 \mathrm{~mm}$ e 3-4 mm de espessura. Nas conexões elétricas foi utilizado cabo de cobre isolado com filme de PVC soldado aos metais envolvidos com seção transversal de 2,5 $\mathrm{mm} 2$. As barras de aço carbono foram fixadas na placa de cobre com resina para solda a frio. Todos os metais foram polidos com escova com fios de aço. Em seguida, foram enxaguados com água destilada, imersos em álcool e secos ao ar. A Figura 1 mostra um esquema do sensor.

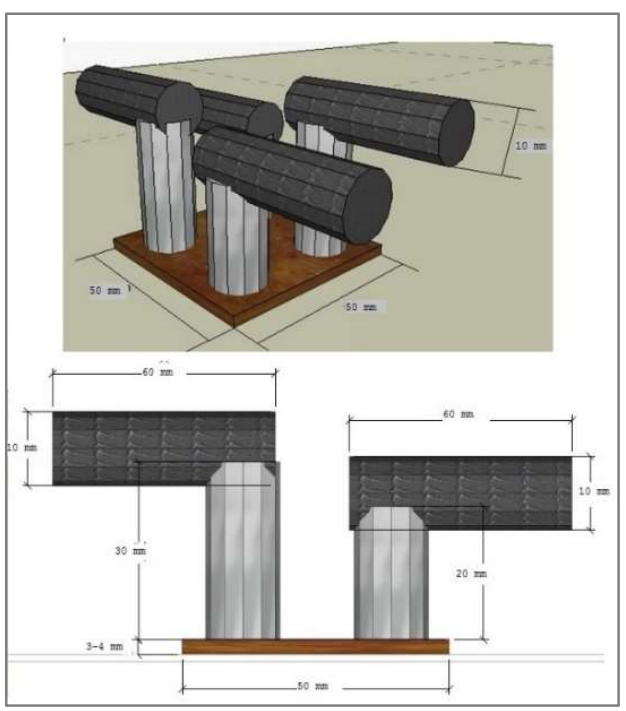

Figura 1: Sensor galvânico confeccionado nos laboratórios da UFPR. Fonte: Autores.

\subsection{Potencial do par (EAR)}

A polarização pode ser definida como o deslocamento de um potencial do seu valor de equilíbrio como resultado de um determinado processo. Com a formação do par galvânico o eletrodo que recebe elétrons (cátodo) passa a sofrer uma polarização catódica. Já o eletrodo que doa elétrons (ânodo) é polarizado anodicamente. A polarização devido à formação de um par galvânico depende da diferença do potencial de equilíbrio irreversível entre os dois eletrodos. Assim quanto mais nobre for um potencial em relação ao outro, isto é quanto maior seja a resistência de corrosão entre eles, mais intensa tende a ser a polarização [9]. Por tanto, é mais ativa quanto maior for esta diferença de potencial [10].

Com base no explicado, o potencial do par ( $E_{P A R}$ ) se determina a partir da diferença entre 0 potencial positivo correspondente ao cátodo e o potencial negativo correspondente ao ânodo conforme a Equação (1).

$$
E_{\text {célula }}=E_{\text {cátodo }}-E_{\text {ânodo }}
$$




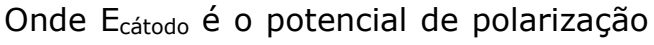
do cátodo e Eânodo é o potencial de polarização do ânodo. Sendo que se $\mathrm{E}^{\circ}$ célula $>0$, então o processo é espontâneo (célula galvânica) e se $E^{\circ}$ célula $<0$, então 0 processo é não espontâneo (célula eletrolítica) [11]

O potencial de polarização de um eletrodo ou metal não pode ser obtido empiricamente e não é possível determinar o valor de potencial de cada eletrodo que forma o par a partir do potencial da célula galvânica já que até mesmo o potencial do par ( $\left.E_{P A R}\right)$ deve ser obtido empiricamente. Sendo Eeq o potencial de equilíbrio irreversível do eletrodo e $\eta$ o sobrepotencial, - qual pode ser anódico ou catódico segundo o tipo de polarização, define-se teoricamente o potencial de polarização como $\mathrm{E}=$ Eeq $\pm \eta$. O potencial de equilíbrio irreversível do eletrodo (Eeq) é atingido quando as velocidades das reações de oxidação e redução são iguais, isto é, a densidade de corrente total (i) se torna densidade de corrente de corrosão ( $i=$ icorr $=\mathrm{ia}=|-\mathrm{ic}|$ ) [9]. Para determiná-lo usa-se um eletrodo de referência. Os potenciais do eletrodo obtidos dessa forma são convencionalmente definidos como potenciais de redução ou potencial de eletrodo padrão e estão em função do meio no qual o eletrodo se encontra imerso.

Entretanto, para fins de desenho de um sensor galvânico um sobrepotencial anódico $\left(\eta_{a}\right)$ se torna um objetivo fundamental já que para conseguir monitorar a corrosão é necessário que o processo de oxidação predomine sobre o processo de redução e a reação entre os metais no sensor seja ativa, ou seja, que predomine a polarização anôdica $\left(i=i_{a}+\right.$ $\left.\left(-\left|i_{c}\right|\right)>0\right)[9]$.

\subsection{Influência da área dos eletrodos na estabilidade de carga no sensor}

A diferença entre o potencial de polarização do ânodo e o potencial de polarização do cátodo será o potencial de referência do sensor galvânico se as condições do meio no qual está inserido o sensor não mudam (E sensor galvânico deve ser capaz de manter estável esse potencial durante um intervalo de tempo finito considerado como o período de vida útil do par. A estabilidade ao longo do tempo do potencial do sensor é influenciada, entre outros fatores, pelas densidades de corrente anódica e catódicas.

Entretanto, as correntes anódicas e catódicas estarão em função da área do ânodo e da área de cátodo respectivamente. Por exemplo, para a mesma densidade de corrente um ânodo de $1 \mathrm{~cm}^{2}$ vai produzir menos corrente total que um ânodo de $10 \mathrm{~cm}^{2}$. O efeito da área no potencial do par segue a tendência da teoria de mistura de potenciais; a qual expõe que um cátodo de grande área pode conduzir o potencial do par para valores mais nobres. De maneira semelhante, um ânodo com uma área grande tende a puxar o potencial do par na direção ativa. Sem embargo, teoricamente o ânodo e o cátodo podem ser feitos de qualquer tamanho já que a relação de área entre o ânodo e o cátodo é a maior responsável por manter um potencial estável do que a área dos eletrodos propriamente dito [11].

Desta forma o período durante o qual o potencial do par se mantém estável pode ser otimizado mudando a relação de área entre o cátodo e o ânodo. Considerando tudo o explicado anteriormente a respeito da influência da relação $A c / A a$ no potencial do par $E_{\mathrm{PAR}}$ a área da placa de cobre que atua como cátodo no sensor galvânico projetado no trabalho foi desenhada de maneira que fosse igual à somatória das áreas dos ânodos que compõem a macrocélula galvânica. Isto ficou evidenciado na Tabela 1.

Tabela 1: Relação entre as áreas.

\begin{tabular}{|c|c|}
\hline Relação Ac/Aa & 0,97 \\
\hline $\mathrm{Ae}$ & $2500 \mathrm{~mm}^{2}$ \\
\hline Af & $942 \mathrm{~mm}^{2}$ \\
\hline$A C$ & $1558 \mathrm{~mm}^{2}$ \\
\hline $\mathrm{Aa}$ & $1607,68 \mathrm{~mm}^{2}$ \\
\hline \multicolumn{2}{|c|}{$\begin{array}{l}\text { Ae: Área exposta da placa } \\
\text { Af: Área dos furos na placa } \\
\text { Ac = (Ae- Af): Área efetiva do cátodo } \\
\text { Aa: Área efetiva do ânodo }\end{array}$} \\
\hline
\end{tabular}

Fonte: Autores

\section{Programa experimental}


Durante a primeira etapa do experimento se analisou o desempenho de dois sensores com a mesma configuração (Figura 1): Sensor A e Sensor B. No artigo são mostrados os resultados obtidos com esse último. O sensor foi submetido a duas condições de exposição diferentes: embebido em uma solução de $\mathrm{Ca}(\mathrm{OH})_{2}$ simulando a solução intercapilar do concreto em condições normais e com adição de 3,5\% de $\mathrm{NaCl}$ em massa a temperatura ambiente para representar um meio contaminado com íons cloretos -. Foram realizadas medidas de Diferença de potencial $\left(E_{P A R}\right)$ entre os metais envolvidos e a Corrente galvânica ( $\left.I_{\text {gal }}\right)$. O peso inicial do sensor era 207,61 g antes de submergilo na solução saturada de $\mathrm{Ca}(\mathrm{OH})_{2} . \mathrm{O} \mathrm{pH}$ da solução e a temperatura ambiente foram mensurados antes de cada leitura com um Medidor de pH LUCA (210) com 90 a 100\% de confiabilidade após calibração.

O experimento compreendeu cinco conjuntos de leituras para cada um dos parâmetros considerados. Cada conjunto de leituras incluiu três testes em média, com exceção do quarto conjunto que só envolveu dois e do terceiro que teve quatro leituras, uma delas de referência. A partir do terceiro conjunto à solução de hidróxido de cálcio $\mathrm{Ca}(\mathrm{OH})_{2}$ foi adicionado o cloreto de sódio $(\mathrm{NaCl})$.

\subsection{Teste do potencial do par galvânico}

Com o intuito de determinar o potencial dos pares do sensor imerso na solução saturada de hidróxido de cálcio com e sem contaminação de cloreto de sódio, foi lida a diferença de potencial dos sensores durante 40 minutos no mínimo em cada leitura. Para isso, usou-se um adquiridor de dados LabVIEW 8.5. Cada um dos quatro pares (ânodo-cátodo) dos sensores projetados gerou uma leitura individual. O software associado ao equipamento registra a diferença de potencial por canal em [V/s]. No processamento dos dados fez-se a conversão das leituras de [V] para [mV] e determinou-se a leitura média em [mV/min.] para cada um dos pares. Em seguida foi analisado se a leitura de diferença de potencial se mantém estável ao longo do tempo e o tipo de reação que indicava (galvânica ou eletrolítica). Considerou-se estável ao longo do tempo o potencial de um par quando a diferença de potencial do par entre as leituras não fosse maior do que $1 \mathrm{mV} / \mathrm{min}$.

\subsection{Teste de corrente galvânica}

A técnica Amperímetro de resistência nula (ZRA) do software EC-Lab é uma aplicação para a medição de corrente galvânica em um par formado por metais diferentes. Para a medição usou-se um potenciostato SP-200. A técnica é usada também para desempenhar alguns tipos de medições de ruído eletroquímico. Consiste em estabilizar a voltagem entre o eletrodo de trabalho (WE) e o contra eletrodo (CE) e medir a corrente e o potencial (Ewe) versus o eletrodo de referência (REF) [12]. Teoricamente, um aumento da corrente galvânica, acompanhado por uma diminuição do potencial de circuito aberto, indica claramente que um nível crítico de íons cloreto foi atingido ou que a frente de carbonatação alcançou as barras do ânodo do sensor $[5 ; 6]$. Entretanto, o valor da corrente galvânica é função dos metais envolvidos no par galvânico. Por tanto, não há uma faixa fixa de valores de corrente galvânica que caracterizam o estado ativo das barras de aço-carbono do ânodo. Isso porque existe uma variedade de interferências na corrente circundante, como a área dos elétrodos, a qualidade do concreto e o teor de íons cloreto no material [13]. Assim sendo, não foi o valor absoluto da corrente o que foi considerado, mas sim a variação de seus valores ao longo do tempo.

\section{Resultados e discussão}

\subsection{Teste do potencial do par}

A Figura 2 resume o comportamento do potencial dos pares do Sensor B durante os 124 dias que esteve imerso na solução. O gráfico está composto pelos valores médios obtidos nas leituras que compõem os cinco conjuntos desenvolvidos. Os valores representados no gráfico são resultado da combinação dos potenciais do cobre e do aço carbono (EAR). A partir dos valores 
obtidos nos pares é possível determinar um valor médio geral para o sensor como um todo em cada leitura. Teoricamente, essa diferença de potencial média pode ser considerada como o potencial de referência do sensor galvânico. Também teoricamente, o sensor galvânico deve ser capaz de manter ou voltar ao valor médio no transcurso do tempo de forma tal que possa ser usado como um potencial de referência confiável se as condições do meio no qual está o sensor não mudam. Porém, já que as leituras individuais dos pares apresentaram variação significativa optou-se por não determinar um valor de potencial para o sensor como um todo e sim considerar cada par de forma individual.

Na Figura 2 observa-se que o par 1 é o único cuja diferença de potencial foi negativa durante $\mathrm{o}$ primeiro conjunto. $\mathrm{O}$ par 2 manteve 0 maior potencial comparando os quatro pares; apresentou valores acima de $250 \mathrm{mV} / \mathrm{min}$. Os pares 3 e 4 apresentaram maior estabilidade que os primeiros variando de 10 a $35 \mathrm{mV} / \mathrm{min}$. No segundo conjunto a diferença de potencial dos pares 2,3 e 4 continuou positiva e o potencial do par 1 virou positivo após a Leitura 1 . Neste caso, os pares apresentaram maior estabilidade durante as três leituras.

Como explicado, durante os dois primeiros conjuntos de leitura o potencial do sensor, em média, foi positivo (reação galvânica) o que indica o predomínio de uma polarização anódica no par. Para fins de projeto de um sensor galvânico, a polarização anódica do sistema se torna um objetivo fundamental já que para conseguir monitorar a corrosão é necessário que a reação entre os metais no sensor seja ativa.

O terceiro conjunto de leituras refere-se ao momento em que foi adicionado 3,5\% em massa de $\mathrm{NaCl}$ na solução aquosa saturada de $\mathrm{Ca}(\mathrm{OH})_{2}$. A leitura de referência (Leitura REF) foi feita 20 minutos antes de adicionar $0 \mathrm{NaCl}$ à solução saturada de $\mathrm{Ca}(\mathrm{OH})_{2}$, nesse momento $\mathrm{O}$ sensor tinha imerso na solução 61 dias. Note-se que a adição de $\mathrm{NaCl}$ provocou uma mudança no potencial dos pares, aumentando drasticamente os valores de potencial dos pares.

$O$ quarto e quinto conjuntos mostram a evolução do potencial do par (E longo do tempo após a adição de $\mathrm{NaCl}$ à solução de hidróxido de cálcio $\mathrm{Ca}(\mathrm{OH})_{2}$. O objetivo desta parte do monitoramento foi entender como os valores de $E_{\text {PAR }}$ evoluem ao longo de tempo de exposição na condição de exposição ao ataque de cloretos. Os valores de potencial dos pares aumentaram consideravelmente no quarto conjunto e mostraram maior estabilidade durante as leituras. No tempo equivalente ao quinto conjunto de leituras, os valores de diferença de potencial ficaram acima dos $200 \mathrm{mV} / \mathrm{min}$.

Esse acréscimo nos valores de $E_{P A R}$ se tornando positivos significa que a macrocélula galvânica é mais reativa de forma tal que o processo de corrosão é espontâneo na presença de íons agressivos permitindo identificá-los rapidamente. Pode-se afirmar que a experiência com o Sensor $B$ de tentar detectar a corrosão por cloretos foi positiva, sendo um indicativo da viabilidade de emprego do sensor de corrosão para estruturas de concreto armado.

\subsection{Teste de corrente galvânica}

A diferença entre o potencial positivo correspondente ao cátodo e o potencial negativo correspondente ao ânodo gera uma corrente elétrica entre os eletrodos da ordem de micros amperes em estado estacionário. Quando o teor de íons cloreto atinge um nível crítico ou a frente de carbonatação alcança as barras do ânodo do sensor, a eletricidade entre os eletrodos aumenta [6]. A Figura 3 resume 0 comportamento da corrente galvânica do Sensor B ao longo dos cinco conjuntos de leituras espaçados no tempo. O gráfico está composto pelos valores médios obtidos em cada leitura. No primeiro conjunto a corrente galvânica no Sensor B foi negativa durante as leituras 1 e 3 . Destaca-se a leitura 2 porque durante a mesma a corrente gerada nos pares 1 e 2 foi positiva. No segundo conjunto se destaca a leitura 1. 


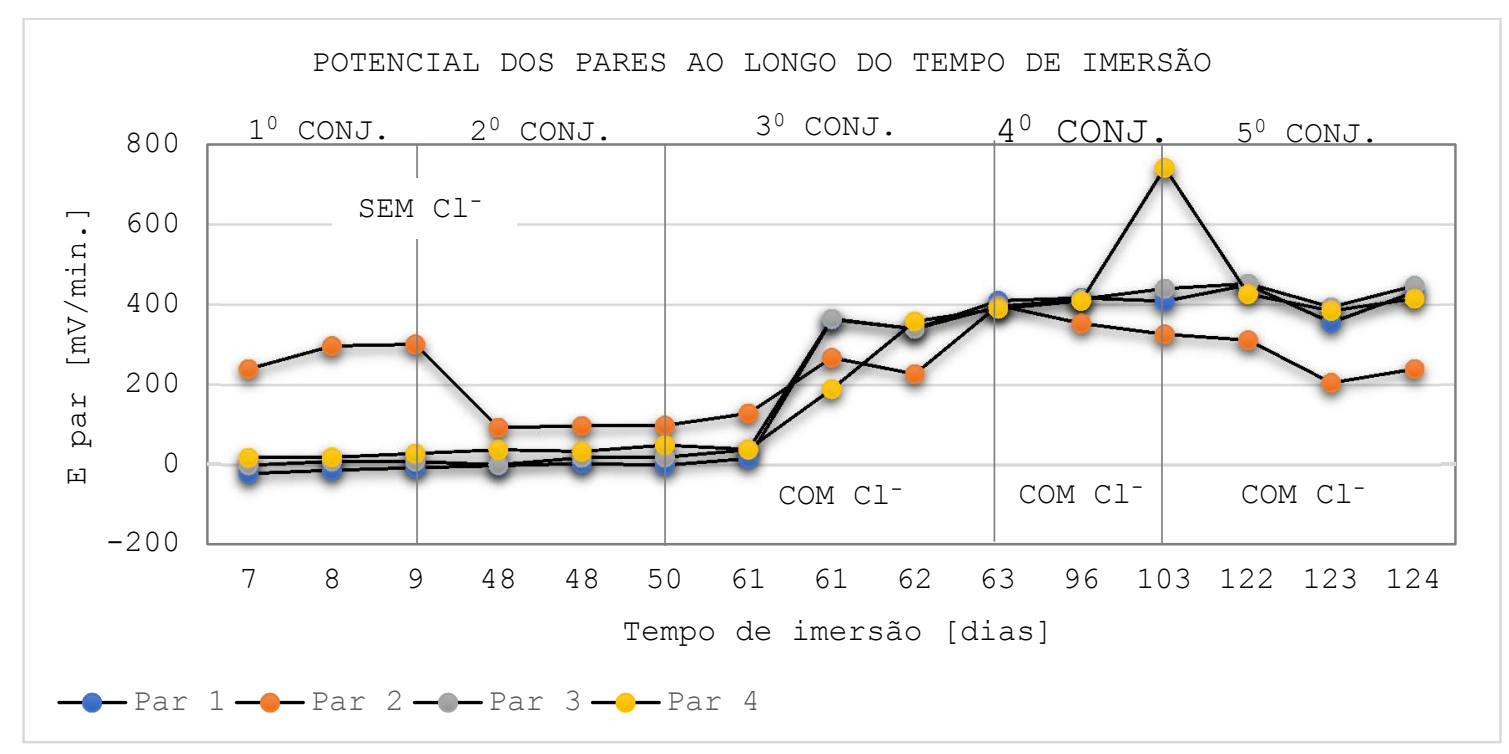

Figura 2: Comportamento do potencial dos pares do Sensor B ao longo do tempo.

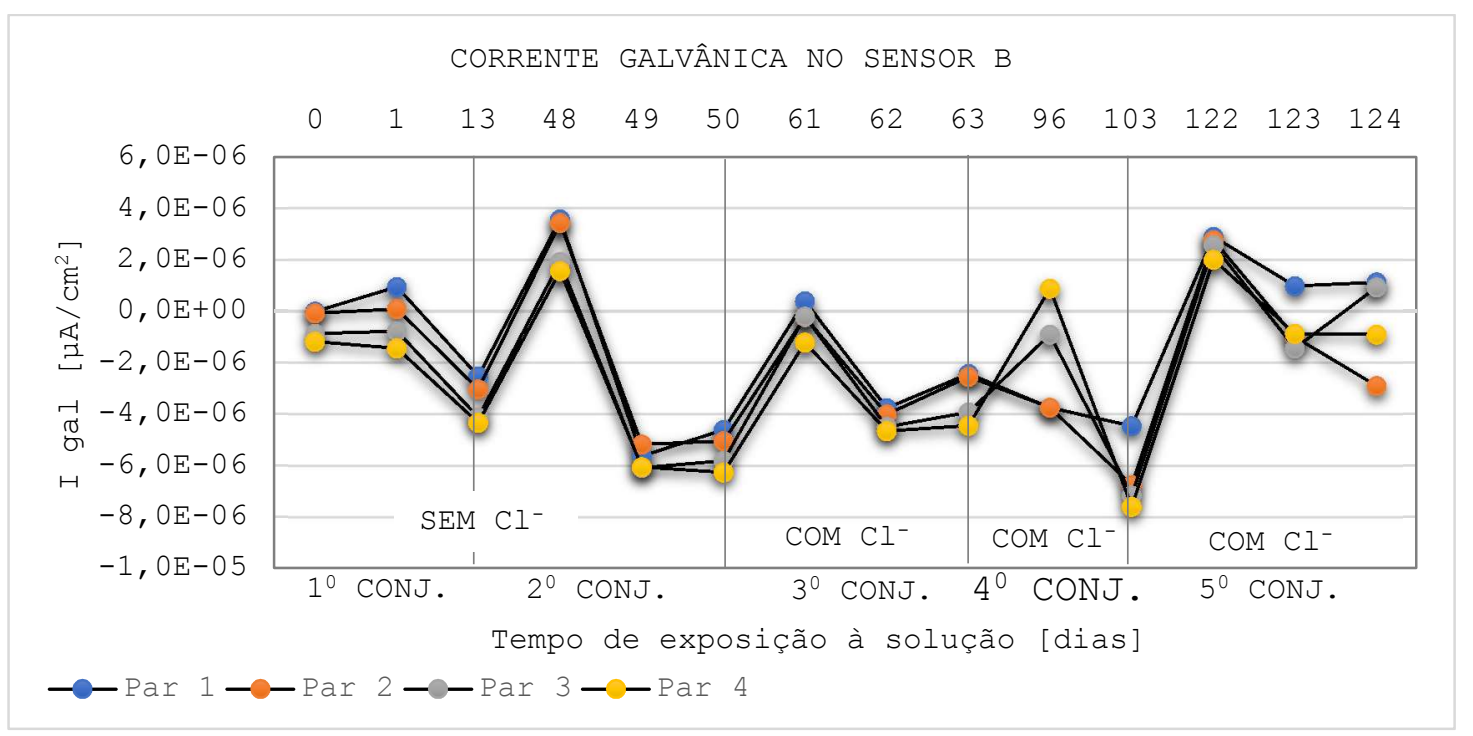

Figura 3: Comportamento da corrente galvânica no Sensor B ao longo do tempo.

Durante esse a corrente gerada em todos os pares foi positiva. Porém, todas as leituras passaram a ser negativa nas leituras 2 e 3. Este comportamento de alternância de $I_{\text {gal }}$ entre valores positivos e negativos indica uma instabilidade do sistema que pode ser causada pelo processo de formação da película passivadora, que segundo Helene (1993) [14] é característico do aço imerso em meio de $\mathrm{pH}$ acima de 12 . Esses resultados de corrente galvânica positivos estão em concordância com os resultados do potencial do par (EPR), que se mostraram positivos indicando uma reação ativa nos pares deste Sensor B já no segundo conjunto de leituras do $E_{\text {PAR }}$ como indica a Figuras 2.

No terceiro conjunto, excetuando a leitura de corrente gerada no par ch00, as demais leituras foram negativas mesmo após a adição de cloreto na solução aquosa saturada de hidróxido de cálcio. É provável que este comportamento seja resultado do valor alto de $\mathrm{pH}$ da solução, o qual ficou acima de 12 ainda durante o terceiro conjunto de leituras, como indica a Figura 4.

Valores negativos de corrente galvânica indicam que o metal ativo está atuando como um cátodo, enquanto o metal mais 
nobre está agindo como ânodo devido ao estado passivo do metal que deveria estar sofrendo oxidação, condição na qual o potencial do metal ativo é superior ao do metal mais nobre [15]. Nessas condições a intensidade da polarização anódica no par é baixa, motivo pelo qual a corrente galvânica permanece pequena.

No quarto conjunto de leituras, o valor de $\mathrm{pH}$ da solução de hidróxido de cálcio é menor do que 11.5 conforme a Figura 4, contudo as leituras de corrente galvânica ficaram negativas. Um dos fatores que pode ter determinado esse comportamento é o baixo teor de oxigênio disponível já que - Sensor B durante todo o experimento ficou totalmente submerso na solução. Segundo Huafu et al. (2015) [16], a pouca disponibilidade de oxigênio pode reduzir a corrente galvânica a zero. Pereira et al. (2009) [17] expõem outro fator plausível: a formação dos óxidos na superfície do eletrodo de trabalho, pode diminuir o valor da $I_{\text {gal }}$ com o tempo, por agir como uma camada que dificulta o progresso da corrosão.

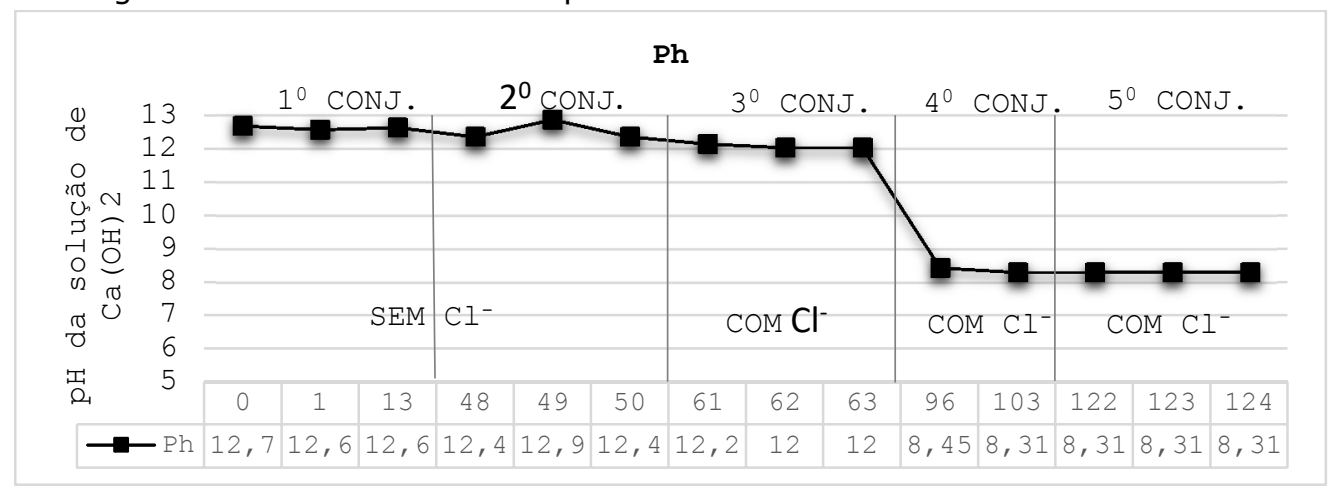

Figura 4: pH da solução saturada de hidróxido de cálcio ao longo do tempo.

Segundo Park et al. (2003) [18], o fato da corrente galvânica diminuir ao longo do tempo, após a quebra da capa de passivação do aço, está intimamente relacionado com o acúmulo de cristais de $\mathrm{Ca}(\mathrm{OH})_{2}$ na superfície do aço. Os cristais de $\mathrm{Ca}(\mathrm{OH})_{2}$ podem restringir uma queda de $\mathrm{pH}$ na superfície de aço. Os cristais proporcionam assim proteção contra a corrosão do aço.

Tanto Park et al. (2003) [18] como Pereira et al. (2009) [17] fazem menção a processos ou fatores que diminuem a área do metal. Os dados de massa registrados no terceiro e quarto conjunto confirmam a perda de área neste caso, a saber $1,49 \%$ e $1,56 \%$ na Leitura 1 e 2 do terceiro conjunto, respectivamente, e $1,66 \%$ no quarto conjunto.

Geralmente, se a área de metal for diminuída, as curvas para ambas as reações associadas (dissolução anódica e redução catódica em metal) se movem para valores inferiores de corrente proporcionais à diminuição da área [18]. Sendo assim, serão necessárias outras leituras para comprovar esse comportamento da corrente e sua correlação com a perda de área.

No quinto conjunto os valores de corrente galvânica de todos os pares se mostram positivos na leitura 1 . 0 valor de corrente lido no par 1 foi positivo durante as três leituras do conjunto. Na leitura 20 par 3 mostra um valor positivo e na leitura 3 o par 4 . De modo geral, isso significa que as leituras de $I_{\text {gal }}$ começam a identificar a atividade de corrosão no sensor, que iniciou no ato de introdução da contaminação do $\mathrm{NaCl}$ na solução em que o sensor está imerso. Comparando estes dados de corrente galvânica com os de Potencial do par (E $\left.E_{P A R}\right)$, pode-se afirmar que $I_{g a l}$ apresenta uma maior inércia para a identificação da atividade de corrosão causada pela contaminação do meio com íns cloretos.

\section{Conclusões}

Esperava-se que 0 sensor se comportasse como um semi-eletrodo de referência capaz de manter ou voltar a um valor médio de potencial no transcurso do tempo. Porém, cada par mostrou um 
comportamento diferenciado. A variação no valor de potencial dos pares resultou ser maior do que $1 \mathrm{mV} / \mathrm{min}$ entre leituras demostrando, portanto, um comportamento instável. Desse modo, deve-se analisar o potencial de cada um dos quatro pares do sensor de forma individual e monitorar a sua alteração.

Mas de forma geral, é possível observar um comportamento padrão. No começo do monitoramento o potencial dos pares foi negativo (reação eletrolítica). A contaminação com $\mathrm{NaCl}$ da solução aquosa saturada com $\mathrm{Ca}(\mathrm{OH})_{2}$ teve um efeito imediato no potencial de todos os pares fazendo com que os valores de potencial dos pares se tornassem positivos (reação galvânica) e com tendência a aumentar ao longo do tempo.

A corrente galvânica também aumentou quando após ter introduzido cloretos na solução aquosa saturada de hidróxido de cálcio, porém, com uma inercia maior do que o potencial do par (E $E_{P A R}$ ) já que as leituras de corrente galvânica continuaram sendo muito baixas. O teste do potencial do par resultou ser mais sensível à contaminação com cloretos do que o teste de corrente galvânica.

Portanto, se faz necessário continuar monitorando os sensores embebidos na solução de hidróxido de cálcio contaminada com 3,5\% de $\mathrm{NaCl}$, em massa, com o intuito de compreender o comportamento da corrente galvânica. A principal limitação do trabalho resultou ser a avaliação dos resultados produto dos inúmeros fatores que influenciam as grandezas mensuradas.

\section{Agradecimentos}

Os autores agradecem ao Laboratório de Materiais e Estruturas da Universidade Federal do Paraná (LAME-DCC-UFPR), ao Programa de Pós-graduação em Engenharia de Construção Civil (PPGECCUFPR), a Coordenação de Aperfeiçoamento de Pessoal de Nível Superior (CAPES) e Conselho Nacional de Desenvolvimento Científico e Tecnológico (CNPq). Também agradecemos ao técnico de laboratório Ricardo Volert pela viabilização da instrumentação que foi necessária para a pesquisa.

\section{Referências}

[1] HOOKS, J. M.; VARMA, S.; PROCTOR, G. D. Proceedings of the National Bridge Management. Inspection and Preservation Conference. 01-02 November del 2011. St. Louis, Mo. Report. U.S. Department of Transportation. Federal Highway Administration (FHWA).

[2] SANTOS, L. O. Monitoramento e ensaio de pontes. In: Congresso Brasileiro de Pontes e Estruturas. 21,22 e 23 de Maio / 2014. Rio de Janeiro. Anais. p 1-14. 21-23.

[3] ALMEIDA, P. A. O.; NETO, G. N. A.; FIGUEIREDO, E. P. Monitoração de estruturas de concreto do livro Concreto: Ciência e tecnologia. In: Concreto: Ciência e Tecnologia. v II. 2011. cap 35. p 1232-1281.

[4] BÄSSLER, R.; PORTELLA, P.D.; PANOSSIAN, Z.; ARAUJO, A. Monitoramento da corrosão em estruturas de concreto: sensor galvânico. Revista Téchne (PINI). Edição 194. p 1-3. Maio / 2013

[5] ARAÚJO, ADRIANA D.; PANOSSIAN, ZEHBOUR; PORTELLA, PEDRO D.; BÄSSLER, RALPH. Monitoramento da corrosão em estruturas de concreto: sensor galvânico. Revista Téchne (PINI). Edição 194. Maio / 2013

[6] ANDRADE, CARMEN; FÉLIX, CARLOS; FIGUEIRAS, HELENA; COUTINHO, JOANA SOUSA. Desempenho do kit-sensor de corrosão na monitorização da durabilidade de estruturas de betão. In: BE2008 - Encontro Nacional Betão Estrutural. 5,6,7 novembro / 2008. Guimarães. Anais. p 1-10.

[7] ANGST, U. AND BUCHLER, M. On the applicability of the Stern-Geary relationship to determine instantaneous corrosion rates in macro-cell corrosion. Materials and Corrosion, 66, No. 10. 2015.

[8] LACERDA, M. D. MARIANNA; MÜLLER RENATA. Uso de sensor de 

armado. Primeira etapa - Testes em solução aquosa.

taxa de corrosão instantânea como técnica de monitoramento da corrosão em estruturas de concreto. Obra24horas. Entrada em: 17/11/2015. Disponível em: <http://www.obra24horas.com.br /artigos/concreto/uso-do-sensorde-taxa-de-corrosao-instataneacomo-tecnica-de-monitoramentoda-corrosao-em-estruturas-deconcreto>. Acesso em: janeiro / 2017.

[9] SOUSA, CALDAS D. CARLOS ALBERTO. Princípios da corrosão eletroquímica. In: Corrosão em estruturas de concreto armado: Teoria, Controle Métodos de Análise. ed. Campus. 2014. cap 2. p 13-34.

[10] FERNANDES,

KETTERMANN. VIVIANE; MARTENDA, PEREIRA. CAROLINE. L. Por que os metais sofrem corrosão? Engenheiro de Materiais. Entrada em: 18 de novembro 2015. Disponível em: $<$ http://engenheirodemateriais.co m.br/tag/pilha-galvanica/> .

[11] PAWLICK, L.A.; STONER, GLEN E.; CLEMEÑA, GERARDO G. Development of na embeddable reference electrode for reinforced concrete structures. Virginia transportation research council. 1998.

[12] EC-LAB. Software: Techniques and Applications. Version 10.1x. Fevereiro, 2011. BioLogic Science Instruments.

[13] M. RAUPACH; P. SCHIESSL. Macrocell sensor systems for monitoring of the corrosion risk of the reinforcement in concrete structures. NDT E INTERNATIONAL. 34(6). 2001. p. 435-442.

[14] HELENE, P. Contribuição ao estudo da corrosão em armaduras de concreto armado. Tese (Livre docência) Escola Politécnica, Universidade de São Paulo. São Paulo, 1993.

[15] PARK, ZIN-TAEK; CHOI, YOON-SEOK; KIM, JUNG-GU; CHUNG, LAN. Development of a galvanic sensor system for detecting the corrosion damage of the steel embedded in concrete structure. Part 2. Laboratory electrochemical testing of sensors in concrete. Cement and Concrete Research, 35. p 1814 1819. 2005.

[16] HUAFU PEI, ZONGJIN LI, JINRUI ZHANG, QIAN WANG. Performance investigations of reinforced magnesium phosphate concrete beams under accelerated corrosion conditions by multi techniques. Construction Building Materials. v 93. p 982994. 2015.

[17] PEREIRA, ELSA V.; FIGUEIRA, RITA B.; SALTA, MARIA MANUELA L.; FONSECA, INÊS TEODORA ELIAS D. A Galvanic Sensor for Monitoring the Corrosion Condition of the Concrete Reinforcing Steel: Relationship Between the Galvanic and the Corrosion Currents. Journal of Sensor. n 9. p 8391-8398. 2009 Disponível em: <www.mdpi.com/journal/sensors $>$.DOI: $10.3390 / \mathrm{s} 91108391$; ISSN 1424-8220. 2009

[18] PARK, ZIN-TAEK; CHOI, YOON-SEOK; KIM, JUNG-GU; CHUNG, LAN. Development of a galvanic sensor system for detecting the corrosion damage of the steel embedded in concrete structure. Part 1. Laboratory tests to correlate galvanic current with actual damage. Cement and Concrete Research, 33. p 2057 2062, 2003. 